\title{
Exérese cirúrgica de adenoma pleomórfico em palato: relato de caso
}

Surgical excision of pleomorphic adenoma in the palate: case report

Escisión quirúrgica de adenoma pleomórfico en el paladar: reporte de caso

Laís Guimarães PINTO ${ }^{1}$

Nathalia Farias Dantas de FIGUEIREDO ${ }^{1}$

Thaynara Cavalcante Moreira ROMÃO ${ }^{1}$

Lucas André Barros FERREIRA²

Murilo Quintão dos SANTOS ${ }^{3}$

Jéssica Amorim Theotonio PEREIRA ${ }^{4}$

Sirius Dan INAOKA

Davi Felipe Neves COSTA ${ }^{5}$

${ }^{I}$ Graduando em Odontologia pelo Centro Universitário de João Pessoa - UNIPÊ, 58053-000 João Pessoa - PB, Brasil

${ }^{2}$ Residente em Cirurgia e Traumatologia Bucomaxilofacial pelo Hospital Universitário Lauro Wanderley - HULW, 58050-585 João Pessoa - PB, Brasil

${ }^{3}$ Especialista em Cirurgia e Traumatologia Bucomaxilofacial pelo Hospital Universitário Lauro Wanderley - HULW, 58050-585 João Pessoa-PB, Brasil

${ }^{4}$ Residente em Odontologia Hospitalar pelo Hospital Universitário Lauro Wanderley - HULW, 58050-585 João Pessoa - PB, Brasil

${ }^{5}$ Cirurgião Bucomaxilofacial do Hospital Universitário Lauro Wanderley - HULW, 58050-585 João Pessoa-PB, Brasil

\section{Resumo}

Introdução: O adenoma pleomórfico (AP) é um tumor de glândulas salivares, misto, benigno composto de células epiteliais e mioepiteliais dispostas em vários padrões morfológicos, demarcadas dos tecidos circundantes por uma cápsula fibrosa. Dentre as glândulas salivares, é predominante nas intraorais com maior frequência no palato. Sua etiopatologia ainda é controversa. Objetivo: Relatar um caso clínico de adenoma pleomórfico localizado em região póstero lateral direito, do palato tratado cirurgicamente. Relato do Caso: Paciente, sexo feminino, 55 anos, foi encaminhada com laudo histopatológico de adenoma pleomórfico. Ao exame físico, foi observou um aumento de volume caracterizado como um nódulo séssil, consistente à palpação, localizado em palato duro direito, com aproximadamente $4 \mathrm{~cm}$ de extensão em seu maior diâmetro, superfície íntegra, coloração normal e indolor. Ao exame tomográfico, imagem unilocular, bem delimitada, com reabsorção óssea local. Como tratamento, optou-se pela exérese cirúrgica da lesão sob anestesia geral, a qual foi realizada sem intercorrências. Paciente atualmente se encontra com 1 ano de pós-operatório sem sinais de recidiva. Conclusão: O tipo de tratamento descrito apresenta excelente prognóstico, com baixas taxas de recidiva.

Descritores: Adenoma Pleomorfo; Cirurgia Bucal; Patologia Bucal.

\section{Abstract}

Introduction: Pleomorphic adenoma (PA) is a benign, mixed, salivary gland tumor composed of epithelial and myoepithelial cells arranged in various morphological patterns, demarcated from the surrounding tissues by a fibrous capsule. Among the salivary glands, it is predominant in intraoral glands with greater frequency on the palate. Its etiopathology is still controversial. Objective: To report a clinical case of pleomorphic adenoma located in the right lateral posterior region of the palate surgically treated. Case Report: A 55-year-old female patient was referred with histopathological report of pleomorphic adenoma. On physical examination, an increase in volume was observed, characterized as a sessile nodule, consistent with palpation, located on the right hard palate, approximately $4 \mathrm{~cm}$ long in its largest diameter, intact surface, normal and painless color. On tomographic examination, unilocular image, well delimited, with local bone resorption. As treatment, we opted for surgical excision of the lesion under general anesthesia, which was performed without complications. Currently, the patient is 1-year post-operative with no signs of recurrence. Conclusion: The type of treatment described has an excellent prognosis, with low rates of recurrence.

Descriptors: Adenoma, Pleomorphic; Surgery, Oral; Pathology, Oral.

\section{Resumen}

Introducción: El adenoma pleomórfico (AP) es un tumor benigno, mixto, de glándulas salivales compuesto de células epiteliales y mioepiteliales dispuestas en varios patrones morfológicos, delimitados de los tejidos circundantes por una cápsula fibrosa. Entre las glándulas salivales, predomina en las glándulas intraorales con mayor frecuencia en el paladar. Su etiopatología sigue siendo controvertida. Objetivo: Informar un caso clínico de adenoma pleomórfico localizado en la región posterior lateral derecha del paladar tratado quirúrgicamente. Caso clínico: Una paciente de 55 años de edad fue remitida con un informe histopatológico de adenoma pleomórfico. En el examen físico, se observó un aumento en el volumen, caracterizado como un nódulo sésil, consistente con la palpación, ubicado en el paladar duro derecho, de aproximadamente $4 \mathrm{~cm}$ de largo en su diámetro más grande, superficie intacta, color normal e indoloro. En el examen tomográfico, imagen unilocular, bien delimitada, con resorción ósea local. Como tratamiento, optamos por la escisión quirúrgica de la lesión bajo anestesia general, que se realizó sin complicaciones. Actualmente, el paciente está 1 año postoperatorio sin signos de recurrencia. Conclusión: El tipo de tratamiento descrito tiene un pronóstico excelente, con bajas tasas de recurrencia.

Descriptores: Adenoma Pleomórfico; Cirugía Bucal; Patología Bucal.

\section{INTRODUÇÃO}

Os tumores das glândulas salivares
constituem cerca de $3 \%$ de todas as neoplasias. A maioria desses tumores é benigna, dos quais cerca de $70 \%$ são adenomas pleomórficos (APs) ${ }^{1}$. O adenoma pleomórfico é um tumor misto benigno, composto de células epiteliais e mioepiteliais dispostas em vários padrões morfológicos, demarcadas dos tecidos circundantes por uma cápsula fibrosa ${ }^{2}$.

Os APs são responsáveis por $45-75 \%$ de todas as neoplasias das glândulas salivares e podem envolver glândulas salivares maiores e menores ${ }^{3}$. A glândula parótida é a glândula salivar mais afetada. Universalmente, $13,9 \%$ a $51,4 \%$ de todos os tumores das glândulas salivares são intraorais e
$34,7 \%$ a $67,1 \%$ deles são benignos. $\mathrm{O}$ tumor ocorre geralmente, na quarta, quinta e sexta décadas de vida e é mais prevalente em mulheres (60\%). Entre as glândulas salivares intraorais, o AP afeta o palato com maior frequência $(42,63 \%)$, seguido pelo lábio $(10 \%)$, mucosa bucal $(5,5 \%)$, área retromolar $(0,7 \%)$ e assoalho de boca ${ }^{4}$.

A etiopatologia desse tumor ainda é controversa, acredita-se que o adenoma pleomórfico se desenvolva a partir de uma mistura de elementos ductais e células mioepiteliais ${ }^{5}$. Geralmente, o adenoma pleomórfico se apresenta clinicamente como uma tumefação de consistência firme, crescimento lento, indolor, recoberta por mucosa 
íntegra e de coloração normal. No entanto, em alguns casos, pode exibir crescimento rápido, ulceração e grande extensão ${ }^{6}$ Sua transformação maligna, resultando em um carcinoma ex-adenoma pleomórfico é citada, podendo ocorrer em cerca de 3 a $4 \%$ dos casos 5 .

O AP faz diagnóstico diferencial com nódulos benignos de glândulas salivares e outros tumores comuns do complexo maxilofacial, bem como neoplasias de potencial maligno como neurofibroma e rabdomiossarcoma. São considerados também diagnósticos diferenciais o lipoma, sarcoma de Kaposi, condiloma acuminata, carcinoma de células escamosas, papiloma oral e goma sifilítica ${ }^{7}$. Sendo assim, a biópsia deve sempre ser realizada.

$\mathrm{O}$ tratamento para o adenoma pleomórfico de glândulas salivares menores consiste na enucleação conservadora. Essa modalidade de tratamento apresenta excelente prognóstico quando realizada a remoção cirúrgica adequada, com baixas taxas de recidiva e rara transformação maligna ${ }^{8}$.

Esse trabalho tem por objetivo relatar um caso clínico de adenoma pleomórfico localizado em região póstero lateral do palato tratado cirurgicamente.

\section{CASO CLÍNICO}

Paciente feoderma, sexo feminino, 55 anos, foi encaminhada ao serviço de Cirurgia e Traumatologia Bucomaxilofacial do Hospital Universitário Lauro Wanderley com laudo histopatológico de adenoma pleomórfico após biópsia incisional realizada previamente. Paciente relatou ausência de doenças de base, mas afirmou que foi tabagista por 20 anos. Ao exame físico extraoral, não foi observada nenhuma alteração relevante. Durante o exame físico intraoral, observou-se uma prótese total maxilar em boas condições sobre patologia diagnosticada. A lesão apresentava-se como aumento de volume, caracterizado como um nódulo séssil, firme à palpação, localizado em palato duro do lado direito, aproximadamente $5 \mathrm{~cm}$ de extensão no seu maior diâmetro anteroposterior, íntegra, indolor sem aspecto inflamatório (Figura 1).

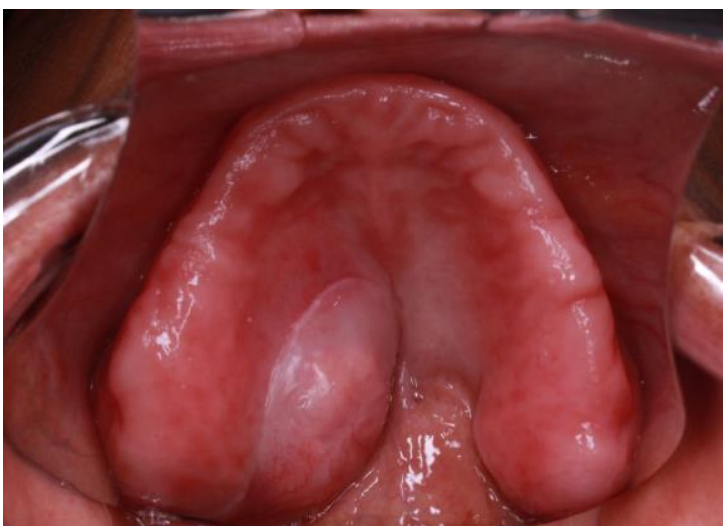

Figura 1: Visão intraoral evidenciando lesão exofítica no palato (lado direito).
Em exame tomográfico de face, verificou-se uma área hipodensa bem delimitada, medindo cerca de 50mm, apresentando reabsorção óssea palatina, causando uma pequena comunicação buconasal (Figura 2). Como tratamento, optou-se pela exérese cirúrgica da lesão sob anestesia geral e intubação nasotraqueal. Foi realizada uma incisão vertical na mucosa adjacente à lesão e em seguida afastamento da mucosa sadia (Figura 3). A ressecção completa do tumor foi obtida (Figura 4), sem intercorrências.

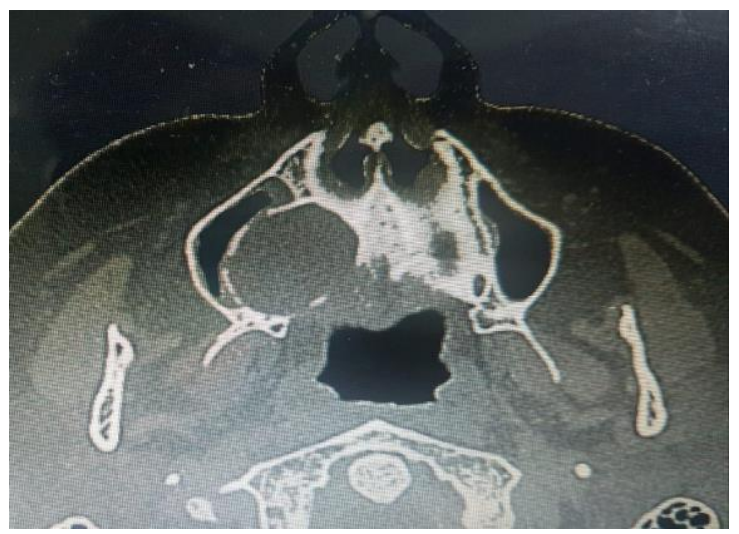

Figura 2: Corte axial de tomografia computadorizada de face apontando lesão osteolítica em região do palato.

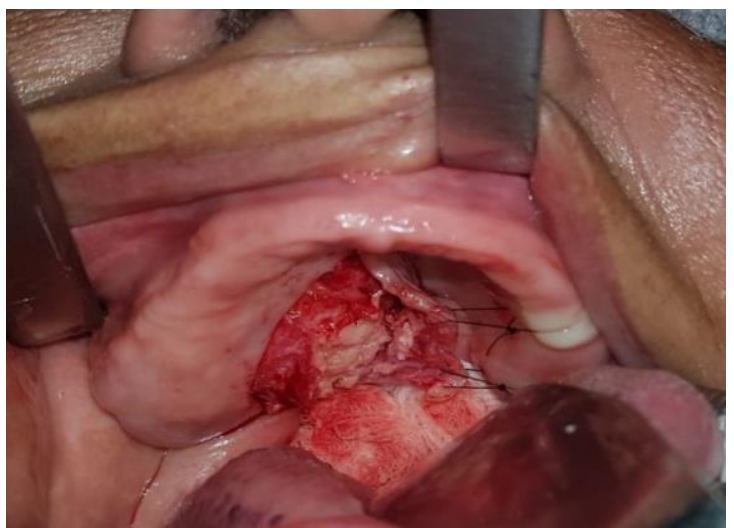

Figura 3: Aspecto cirúrgico após o descolamento da mucosa palatina.

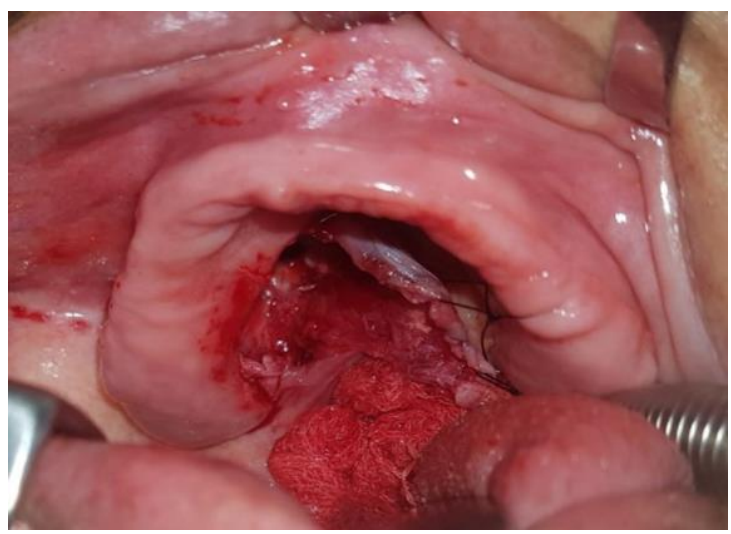

Figura 4: Aspecto cirúrgico após a ressecção completa do tumor.

A amostra foi enviada para análise histopatológica (Figura 5), a fim de confirmar o resultado histopatológico prévio. A síntese da ferida foi dada através de sutura simples com fio de Vycrilß 4.0 (Figura 6). No exame macroscópico, apresentou múltiplos fragmentos teciduais 
irregulares, ora pardo-acinzentados, ora pardoesbranquiçados, elásticos, permeados por líquido vinhoso, medindo em conjunto 5,0 x 4,3 x 1,0 cm. A conclusão histopatológica foi de adenoma pleomórfico, com ausência de sinais de malignidade. A paciente encontra-se com um ano de acompanhamento pós-operatório, sem sinais de recidiva até o momento (Figura 7 ).

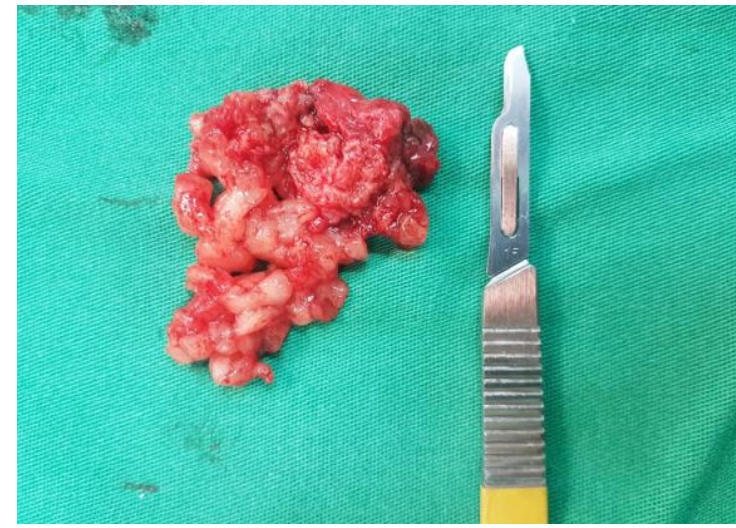

Figura 5: Visão macroscópica da lesão após exérese.

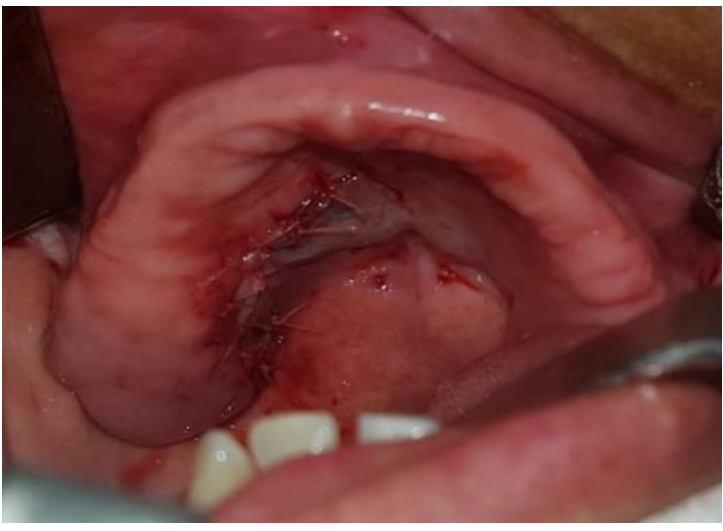

Figura 6: Sutura.

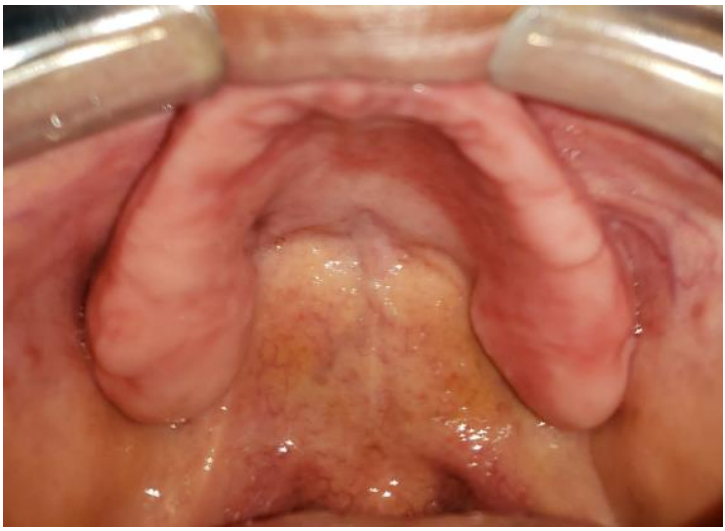

Figura 7: Visão intraoral do pós-operatório de 1 ano.

\section{DISCUSSÃO}

Alterações tumorais em glândulas salivares são de grande interesse para a Cirurgia Bucomaxilofacial por não ocorrerem em outras partes do corpo humano. O adenoma pleomórfico é a neoplasia benigna de glândula salivar mais comum reportado pela literatura, sendo as glândulas salivares menores do palato o local mais comum para o surgimento do AP intraoral. Bem como observado no estudo demográfico de Takahashi et al. ${ }^{9}$, em que na avaliação de 200 tumores intraorais, 124 foram diagnosticados como adenomas pleomórficos ${ }^{8,9}$.

As mulheres constituem o sexo mais afetado pelo AP. De acordo com uma pesquisa realizada em São Luís - MA, em 2008, de 138 registros do AP 85 pertenciam ao sexo feminino. Corroborando, com um estudo retrospectivo onde foi observado também maior incidência no sexo feminino, com relação de 3, $5: 1^{10,11}$. O AP pode apresentar diagnóstico diferencial com outros tipos tumores benignos do complexo maxilofacial, como rabdomiossarcoma, neurofibroma, lipoma e linfoma. Em adicional a esses, são considerados também diagnósticos diferenciais o carcinoma de células escamosas, sarcoma de kaposi, condiloma acuminata, goma sifilítica e papiloma oral ${ }^{7,8}$.

$\mathrm{O}$ adenoma pleomórfico se manifesta como uma massa indolor, de crescimento lento, persistindo por anos. Seus sintomas sofrem influência dependendo da localização ${ }^{1,12}$. No caso reportado, a lesão foi encontrada no palato duro do lado direito, acarretando complicações como a reabsorção do processo palatino direito que ocasionou uma pequena comunicação buconasal. Outros sintomas que podem ser encontrados em AP em glândulas salivares menores são disfagia, dispneia e dificuldade de mastigar, não relatadas pela paciente atendida.

O tratamento mais indicado é a excisão local ampla da lesão, em conjunto com biópsia para confirmação da hipótese diagnóstica ${ }^{12}$. Lesões recidivantes podem acontecer quando a extensão microscópica está além da cápsula ou por ruptura da cápsula durante a exérese cirúrgica ${ }^{13}$. Nesse caso clínico, não foi removida a mucosa sob a lesão.

Para a possibilidade de transformação maligna do AP, foi proposta a mutação de famílias de fatores de risco, que estão envolvidas no início, avanço e vascularização dos carcinomas. São elas as proteínas PDGF-A, PDGF-B, PDGF-R alfa, FGF-2, BEK E FIg, que estariam envolvidas no crescimento livre de células neoplásicas ${ }^{14}$. A possibilidade de transformação maligna é minimizada quando a remoção da lesão é realizada o quanto antes. Como pôde-se observar no caso clínico de Maia et al., em que o paciente foi tratado cirurgicamente após 6 meses de evolução da lesão e posteriormente, apresentou cicatrização e ausência de recidivas em acompanhamento durante um ano ${ }^{15}$. No caso clínico referido neste relato, após um ano de acompanhamento pós-operatório a paciente não apresenta sinais de recidivas para AP. Não foram encontrados na literatura, casos de transformação maligna de AP, originados do uso de cigarros, levando em consideração que a paciente fumou durante 20 anos.

CONSIDERAÇÕES FINAIS

O tipo de tratamento descrito apresenta 
excelente prognóstico, com baixas taxas de recidiva e rara transformação maligna. Paciente encontra-se em acompanhamento pós-operatório de 1 ano, até o momento, não houve sinais de recorrência da lesão.

Sendo assim, o diagnóstico precoce das alterações das glândulas salivares se torna importante para a realização de um tratamento mais conservador e uma melhora no prognóstico.

\section{REFERÊNCIAS}

1. Sharma A, Deshmukh S, Shaikh A, Dabholkar J. Pleomorphic adenoma of the minor salivary gland of the cheek. Singapore Med J. 2013;54(9):e183-4.

2. Arumugam P, Christopher PJ, Kumar S, Kengasubbiah S, Shenoy V. Pleomorphic adenoma of the palate: a. case report. Cureus 11(3):e4308.

3. Khan MN, Raza SS, Hussain Zaidi SA, Haq IU, Hussain AK, Nadeem MD, Farid K. Pleomorphic Adenoma Of Minor Salivary Glands. J Ayub Med Coll Abbottabad. 2016;28(3):620-22.

4. Chaturvedi M, Jaidev A, Thaddanee R, Khilnani AK. Large Pleomorphic Adenoma of Hard Palate. Ann Maxillofac Surg. 2018;8(1):124-126.

5. Oliveira LJ, Castro HHO, Leão PLR, Leal RM, Horta MCR, Souza PEA. Tratamento de adenoma pleomórfico em palato: relato de 2 casos e revisão de literatura. Rev Port Estomatol Med Dent Cir Maxilofac. 2016;57(1):55-61.

6. Porto DE, Cavalcante JR, Cavalcante Júnior JR, Costa MCF, Pereira SM. Adenoma Pleomórfico de Parótida - Relato de Caso. Rev cir traumatol bucomaxilo-fac. 2014;14(2):15-18;

7. Melo MNB, Nogueira Neto JN, Souza SR, Dultra FKAA, Dultra JA. Adenoma pleomórfico em lábio superior: Relato de caso. Rev. cir. traumatol. bucomaxilo-fac.2016;16(2):40-3.

8. Erdem MA, Cankaya AB, Güven G, Olgaç V, Kasapoğlu C. Pleomorphic adenoma of the palate. J Craniofac Surg. 2011;22(3):1131-4.

9. Takahashi H, Fujita S, Tsuda N, Tezuka F, Okabe H. Intraoral minor salivary gland tumors: a demographic and histologic study of 200 cases. Tohoku J Exp Med. 1990;161(2):111-28.

10. Loiola RF, Matos FR, Nonaka CFW, Lopes FF, Cruz MCFN. Perfil epidemiológico das neoplasias de glândulas salivares diagnosticadas em São LuísMA. J Bras Patol Med Lab. 2009;45(5):413-20.

11. Tiago RSL, Castro GA, Ricardo LAC, Bühler RB, Fava AS. Adenoma pleomórfico de parótida: aspectos clínicos, diagnósticos e terapêuticos. Rev Bras Otorrinolaringol. 2003;69(4):485-89.

12. Khan MN, Raza SS, Hussain Zaidi SA, Haq IU, Hussain AK, Nadeem MD, Farid K. Pleomorphic Adenoma Of Minor Salivary Glands. J Ayub Med Coll Abbottabad. 2016;28(3):620-22.

13. Soares AB, Demasi APD, Altemani A, Araújo VC. Increased mucin 1 expression in recurrence and malignant transformation of salivary gland pleomorphic adenoma. Histopathology. 2011; 58(3):377-82.

14. Soares AB, Demasi AP, Tincani AJ, Martins AS, Altemani A, de Araújo VC. The increased PDGF-A, PDGF-B and FGF-2 expression in recurrence of salivary gland pleomorphic adenoma. J Clin Pathol. 2012;65(3):272-77.

15. Maia FPA, Oliveira PRK, Santos JVQM, Costa DFN, Andrade ESS. Abordagem minimamente invasiva para tratamento de adenoma pleomórfico em palato: caso clínico. Rev Cir Traumatol Bucomaxilofac. 2019;19(3):21-4.

\section{CONFLITO DE INTERESSES}

Os autores declaram não haver conflitos de interesse.

\section{AUTOR PARA CORRESPONDÊNCIA}

\section{Laís Guimarães Pinto}

Centro Universitário de João Pessoa - UNIPÊ

58053-000 João Pessoa - PB, Brasil

Email: lais_gp@hotmail.com
Submetido em 26/04/2020

Aceito em 28/07/2020 